INPLASY

PROTOCOL

To cite: Sun et al. Systemic immune inflammation index is a prognostic factor for small cell lung cancer: a systematic review and meta-analysis. Inplasy protocol 2021120083. doi:

10.37766/inplasy2021.12.0083

Received: 18 December 2021

Published: 18 December 2021

Corresponding author:

Muzhen Sun

sunmzi@126.com

Author Affiliation:

Anhui University of Science andTechnology Medical

college.

Support: None.

Review Stage at time of this submission: Preliminary searches.

Conflicts of interest:

None declared.

\section{Systemic immune inflammation index is a prognostic factor for small cell lung cancer: a systematic review and meta-analysis}

\author{
Sun, M1; Cao, M2 Zhang, Y3; Kang, $\mathrm{X}^{4}$.
}

Review question / Objective: Compared with a low level of systemic immune inflammation index, does a high level of systemic immune inflammation index increase the risk of poor prognosis in patients with small cell lung cancer?

Condition being studied: Lung cancer has been one of the primary causes of cancer-related mortality globally. In one subtype of lung cancer, small cell lung cancer(SCLC) is a neuroendocrine lung tumor characterized by high aggressiveness, high recurrence, and low survival rate. As an essential bridge between inflammation and cancer, inflammatory factors may be related to tumor risk and survival. The systemic immune inflammation index(SII) is a safe, economical and convenient inflammation index that can be used to assess the prognosis of patients. The purpose of this meta-analysis was to analyze the relationship between systemic immune inflammation index and prognosis in smallcell lung cancer patients.

INPLASY registration number: This protocol was registered with the International Platform of Registered Systematic Review and Meta-Analysis Protocols (INPLASY) on 18 December 2021 and was last updated on 12 January 2022 (registration number INPLASY2021120083).

\section{INTRODUCTION}

Review question / Objective: Compared with a low level of systemic immune inflammation index, does a high level of systemic immune inflammation index increase the risk of poor prognosis in patients with small cell lung cancer?
Condition being studied: Lung cancer has been one of the primary causes of cancerrelated mortality globally. In one subtype of lung cancer, small cell lung cancer(SCLC) is a neuroendocrine lung tumor characterized by high aggressiveness, high recurrence, and low survival rate. As an essential bridge between inflammation and cancer, inflammatory factors may be 
related to tumor risk and survival. The systemic immune inflammation index(SII) is a safe, economical and convenient inflammation index that can be used to assess the prognosis of patients. The purpose of this meta-analysis was to analyze the relationship between systemic immune inflammation index and prognosis in small-cell lung cancer patients.

\section{METHODS}

Search strategy: Records identified from database screening PubMed, Web of Science, ScienceDirect, Cochrane Library, Embase, and Google Scholar from inception to July 2021. The search strategy was performed with the following items: ("Small Cell Lung Cancer" or "SCLC") AND (SII OR "Systemic Immune Inflammation Index"). The publication language had no limitations in this search.

Participant or population: Small cell lung cancer patients.

Intervention: Small cell lung cancer patients with high levels of SII.

Comparator: Small cell lung cancer patients with low levels of SII.

Study designs to be included: Studies include randomised controlled trials, cohort studies, case-control, retrospective and prospective studies.

Eligibility criteria: Inclusion criteria: (1) Study used cytology or pathology to diagnose SCLC;(2) Study measured the counts of neutrophils, platelets, lymphocytes and gave the value of SII; (3)Study provided overall survival and/or progression-free survival for survival analysis; (4) Study provided the hazard ratio (HR) and the 95\% confidence interval; (5)Study reported the relationship between SII and SCLC. Exclusion criteria:(1) Summary, case report, meeting summary, or letter to the editor;(2) Special specimens, unclear data, repeated publications, and studies without full text.
Information sources: PubMed, ScienceDirect, Embase, China National Knowledge Infrastructure (CNKI) and Wanfang literature databases

Main outcome(s): The main result of the current meta-analysis is a poor prognosis for patients with small cell lung cancer.To investigate the correlation between the amount of SII and SCLC prognosis, the pooled $\mathrm{HR}$ and $95 \% \mathrm{Cl}$ were determined. The correlation between the clinical characteristics of SCLC and SII was assessed using odds ratios (ORs) and $95 \%$ Cl.

Data management: Two reviewers independently assessed and incorporated the following items from each study: first author's name, year of publication, country, gender, age, study design, recruitment time, disease stage, follow-up time, treatment method, the cut-off value of SII, survival analysis, $\mathrm{HR}$, and $95 \% \mathrm{Cl}$.

Quality assessment / Risk of bias analysis: The Newcastle-Ottawa Scale (NOS) was used to evaluate the quality of the included studies.

Strategy of data synthesis: The software utilized was Stata 15.0 MP to conduct a meta-analysis of the current research. To investigate the correlation between the amount of SII and SCLC prognosis, the pooled $\mathrm{HR}$ and $95 \% \mathrm{Cl}$ were determined. The correlation between the clinical characteristics of SCLC and SII was assessed using odds ratios (ORs) and 95\% $\mathrm{Cl}$. The heterogeneity of the studies was determined using the Cochran $Q$ chisquare test and the Cochrane-I2 statistic. The fixed-effects model $(12 \leq 50 \%$ and $P$ $\geq 0.05$ ) was used without significant heterogeneity. On the contrary, a randomeffects model would be adopted (12>50\% or $P<0.05)$ and conducted subgroup analysis to determine the likely cause of heterogeneity. A sensitivity analysis was carried out to determine if the findings of single research influenced the overall result. Begg's andEgger's test evaluates publication bias to ensure the reliability of 
the results. The result is statistically significant if the $\mathrm{P}$-value $<0.05$.

Subgroup analysis: Subgroup analysis of OS and SII by region, sample size, disease stage, treatment method, SII cut-off value and NOS score.

Sensitivity analysis: Sensitivity analysis of HR combined with overall surviva and progression-free survival.

Language: The publication language had no limitations in this search.

Country(ies) involved: China and Turkey.

Keywords: small cell lung cancer; systemic immune inflammation index; prognosis; meta-analysis.

Contributions of each author:

Author 1 - Muzhen Sun.

Author 2 - Mei Cao.

Author 3 - Yulian Zhang.

Author 4 - Xipeng Kang. 